\title{
Rapid Determination of Rice Cultivar Responses to the Sheath Blight Pathogen Rhizoctonia solani Using a Micro-Chamber Screening Method
}

\author{
Y. Jia, United States Department of Agriculture-Agricultural Research Service (USDA-ARS) Dale Bumpers Na- \\ tional Rice Research Center (DB NRRC), Stuttgart, AR 72160; F. Correa-Victoria, International Center for Tropical \\ Agriculture, AA6713, Cali, Colombia; A. McClung, USDA-ARS DB NRRC, Stuttgart; L. Zhu, G. Liu, and \\ Y. Wamishe, Rice Research and Extension Center, University of Arkansas, Stuttgart 72160; J. Xie, Jiangxi Academy \\ of Agricultural Sciences, Nanchang 330200, P. R. China; M. A. Marchetti and S. R. M. Pinson, USDA-ARS Beau- \\ mont, TX 77713; J. N. Rutger, USDA-ARS DB-NRRC, Stuttgart; and J. C. Correll, University of Arkansas, Fa- \\ yetteville 72701
}

\begin{abstract}
Jia, Y., Correa-Victoria, F., McClung, A., Zhu, L., Liu, G., Wamishe, Y., Xie, J., Marchetti, M. A., Pinson, S. R. M., Rutger, J. N., and Correll, J. C. 2007. Rapid determination of rice cultivar responses to the sheath blight pathogen Rhizoctonia solani using a micro-chamber screening method. Plant Dis. 91:485-489.

An accurate greenhouse screening method has not been developed previously to identify host response to sheath blight disease caused by Rhizoctonia solani Kühn that causes significant economic losses in rice yield worldwide. The unavailability of a robust screening system in the greenhouse has made it difficult to quantify disease reactions to $R$. solani, and has hampered studies on the genetics of resistance and plant breeding efforts to improve resistance. In an effort to develop a standardized laboratory micro-chamber screening method to quantify resistance to $R$. solani in rice, five rice cultivars, representing a wide range of observed disease reactions under field conditions, were examined in a blind inoculation test at three locations (Arkansas, Texas, and Colombia). Rice seedlings were inoculated at the three- to four-leaf stage with potato dextrose agar plugs containing mycelium and then covered with a 2- or 3-liter transparent plastic bottle for maintaining high humidity after inoculation. Two cultivars, Jasmine 85 and Lemont, that consistently have shown the highest and lowest levels of resistance, respectively, in previous field and greenhouse studies, were used as standards. Concurrent field experiments in Arkansas and Texas also were performed to compare the greenhouse disease ratings with those observed under field conditions. Overall, the relative disease ratings of the seven test cultivars were consistent between test locations and with field evaluations. Thus, the micro-chamber screening method can be used as an effective approach to accurately quantify resistance to the sheath blight pathogen under controlled greenhouse conditions and should help expedite the selection process to improve resistance to this important pathogen.
\end{abstract}

Additional keywords: Oryza sativa

Sheath blight disease caused by the soilborne necrotrophic fungal pathogen Rhizoctonia solani Kühn (teleomorph: Thanatephorus cucumeris) is one of the most destructive rice (Oryza sativa L.) diseases. Disease epidemics often are favored by intensive production practices that include: deployment of semidwarf or leafy rice cultivars, use of high nitrogen inputs, and high seeding rates that encour-

Corresponding author: Y. Jia

E-mail: yjia@spa.ars.usda.gov

Accepted for publication 19 December 2006.

doi:10.1094/PDIS-91-5-0485

This article is in the public domain and not copyrightable. It may be freely reprinted with customary crediting of the source. The American Phytopathological Society, 2007. age lush canopy growth $(5,13)$. In the United States, sheath blight disease has been reported to cause yield losses up to $50 \%$ under environmental conditions favoring disease development $(8,9,12)$.

Developing sheath-blight-resistant cultivars through conventional breeding has reached only limited success due to the lack of resistant adapted germplasm, the limited efficiency and effectiveness of available screening methods, and the polygenic nature of the resistance. For breeding purposes, replicated inoculated field nurseries are the most common means for evaluating overall responses to the pathogen. The field screening method requires more than $30 \mathrm{~g}$ of seed per genotype, large amounts of inoculum, high humidity conditions, and approximately 4 to 5 months to complete field evaluations and summarize the results. Disease ratings in the field are labor intensive and involve taking multiple assessments on disease severity from where the infections are initiated (the flood water level) up through the top of the canopy. Plot ratings are a visual average of the many plants observed in a plot. Results often are confounded by many variables, including fluctuations in environmental conditions and distribution of inoculum. Thus, plots are replicated in different years or locations because disease severity often is confounded by plant height, maturity date, plant architecture, and small differences in observed response are genetically significant, given that host resistance is a polygenic trait, controlled by several genes with relatively small individual effect $(7,11,18)$.

A rapid and reproducible screening system that reduces environmental effects and is not confounded by plant phenotype is essential for quantifying resistance to the pathogen and helping to expedite the selection process to improve resistance. Furthermore, a more robust screening effort is critical to develop a better understanding of the genetics of resistance in rice. To date, a number of systems have been utilized to facilitate breeding for improved resistance to $R$. solani. These assays involved placing sclerotia, hyphae, or infested rice straw or grain on detached leaves or sheaths in vitro or inside the sheath, at the base of rice plants in a growth chamber or greenhouse $(2,3,8,14$ 16). Even though these methods have been useful for some applications, they have not been standardized or widely adopted due to their inherent variability.

The objectives of this study were to develop a standardized robust screening method in the greenhouse to evaluate rice for resistance to $R$. solani and evaluate the reproducibility of the method at different locations.

\section{MATERIALS AND METHODS}

Plant materials and locations. All rice cultivars that can be grown in Arkansas and Texas with known disease reactions to 
the sheath blight pathogen were used in a blind evaluation test (entry 1, 'Cypress;' entry 2, 'Rosemont;' entry 3, 'Pecos;' entry 4, 'Cocodrie;' and entry 5, 'TeQing'). Because no source of complete resistance has been identified in cultivated rice, cultivar susceptibility in this study was measured relative to the moderately resistant standard, 'Jasmine 85,' and to 'Lemont,' a highly susceptible standard $(1,6)$. The cultivars were coded and seed from a single source (to ensure genetic identity and minimize seed-quality effects on the study) was delivered to all test locations: Dale Bumpers National Rice Research Center (DB NRRC) Arkansas; International Center for Tropical Agriculture, Colombia, and the United States Department of Agriculture-Agricultural Research Service (USDA-ARS), Texas.

Pathogen isolates. The isolate of $R$. solani (RR0140-1), which had a slowergrowing phenotype than most typical wildtype isolates (Y. Wamishe and Y. Jia, unpublished), was selected among 102 isolates collected from rice fields in Arkansas because it produced consistent disease reactions on standard cvs. Jasmine 85 and Lemont in Arkansas. An isolate recovered from symptomatic plants in Texas (TMR-1) and Colombia (Tol 2399-1) was used in the screening tests at these locations. Inoculum was grown by placing sclerotia on potato dextrose agar (PDA) (Sigma-Aldrich, St. Louis) containing tetracycline $(0.005 \%$, $\mathrm{wt} / \mathrm{vol}$ ) and then transferring mycelium to fresh medium to produce active mycelia. Each isolate was maintained on PDA in petri dishes at 27 to $30^{\circ} \mathrm{C}$.

Inoculum for the present study consisted of round disks ( $1 \mathrm{~cm}$ in diameter) that were excised from a 3-day-old mycelia culture grown on PDA containing tetracycline $(0.005 \%, \mathrm{wt} / \mathrm{vol})$ in petri dishes. Each petri dish contained $20 \mathrm{ml}$ of PDA. Mycelial discs with a diameter of approximately 7 $\mathrm{mm}$ were excised from similar radii in petri dishes to maintain uniformity in virulence. Multiple plates were required to provide sufficient inoculum for each replication.

Micro-chamber method. The microchamber method initially was conceived and preliminarily developed by Dr. Ali at the Bangladesh Rice Research Institute, Rahshahi, Bangladesh. The microchambers were made by removing the screw caps and bottoms of 2- or 3-liter soft drink bottles. Preliminary studies (Y. Jia, unpublished) indicated that the color of the bottle (clear or green) was not critical to the results. Before potting, rice seeds were germinated on moist filter paper in petri dishes for 2 days at $30^{\circ} \mathrm{C}$. Three seedlings of similar germination rate were selected and planted at least $3 \mathrm{~cm}$ apart in a $15-\mathrm{cm}-$ diameter plastic pot filled with soil obtained from each location's fields. Soil was first steam sterilized to ensure that the study was not confounded by the presence of soilborne $R$. solani inoculum. Pots were placed in flats containing shallow water (approximately $5 \mathrm{~cm}$ ) and were watered through holes near their bottoms. Pots were placed in a greenhouse until the three- to four-leaf stage before inoculation. Pots were arranged in a completely random design with the susceptible Lemont and moderate-resistant Jasmine 85 serving as standards. Each cultivar was represented by three replicate plants per pot per replication. The study was repeated in time in each of the locations. Each seedling was inoculated with a round mycelial disk placed and pressed up to the base of the stem, assuring that mycelium was in contact with the plant. After inoculation, each pot was covered with a soft drink bottle that was pushed down into the soil to create a seal that allowed the bottle to serve as a micro-humidity chamber. The cap was left off the bottle. Day and night greenhouse temperate averaged 34 and $24^{\circ} \mathrm{C}$, respectively, at both locations in the United States and 30 and $21^{\circ} \mathrm{C}$, respectively, in Colombia. The inoculated pots were maintained in trays containing water in the greenhouse. Relative humidity was always above $80 \%$ within the bottles at all locations.

Lesion development was monitored starting 3 days post inoculation (dpi). At all locations, the plants were evaluated for disease reactions approximately 7 to $10 \mathrm{dpi}$ or when Lemont exhibited a highly susceptible reaction. Diseased seedlings were cut at the ground line and the length of the lesion was measured along with the length of the culm using an electronic digital caliper (ISO 17025; VWR, West Chester PA) or ruler scale $(\mathrm{mm})$. A disease index was calculated as lesion length divided by plant height and multiplied by 9 . Sheath blight micro-chamber inoculations were repeated twice in all locations.

Field experiments. The seven cultivars used in the micro-chamber screening test were evaluated under field conditions using $R$. solani isolate RR0140-1 in Stuttgart, AR and TMR-1 in Beaumont, TX. The Stuttgart, AR field studies were planted in May 2005 using a randomized complete block design with four replications. Each three-row plot was bordered with the susceptible cv. Cocodrie to minimize variability between adjacent cultivars. The $R$. solani inoculum was prepared based on the following procedure. Dry sweet corn (40 g) and dry rye grass grain $(30 \mathrm{~g})$ were mixed in 1 liter of water and autoclaved for 45 min twice. Mycelial plugs were incubated in the mixture for 4 to 5 days, and then transferred to a paper bag for producing sclerotia, which were ground and used as inocula. In Beaumont, TX, field plots again consisted of three rows $2.4 \mathrm{~m}$ in length, with $18 \mathrm{~cm}$ between rows and between plots. Texas plots were not bordered by Cocodrie, but were next to each other. The Texas study consisted of eight replications. Inoculum was an $R$. solani-infected 4:1 mixture of rice hulls: paddy (rough) rice autoclaved twice for $2 \mathrm{~h}$ each before infecting. $R$. solani was allowed to grow throughout the mixture for 3 days before it was sprinkled by hand over all field plots. In both locations, rice plants were inoculated at the first internode stage and disease reactions were observed in the center row of the plot approximately 30 days after heading, and showed that ratings at this point in maturity was correlated with disease resistance. Disease was evaluated as visual disease index using a 0 -to- 9 scale, where 0 to 4 was resistant and $>4$ was susceptible.

Data analysis. The statistical analysis of the disease response was based on a completely randomized design of seven cultivars inoculated with subcultures of a field
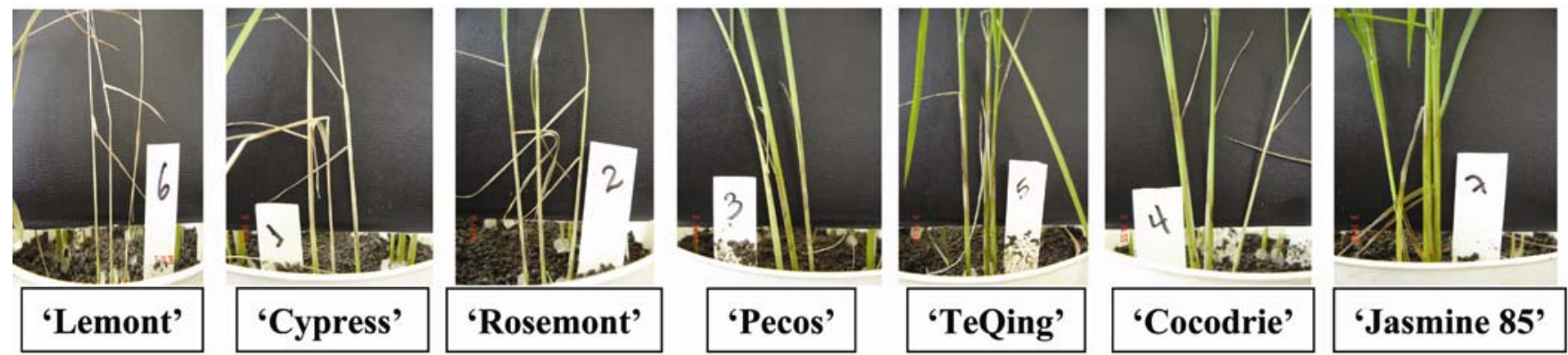

Fig. 1. Symptoms of sheath blight disease detected using micro-chamber method. From left to right: disease reactions of Lemont, susceptible standard; entry 1, Cypress; entry 2, Rosemont; entry 3, Pecos; entry 5, TeQing; entry 4, Cocodrie; and Jasmine 85, moderately resistant standard. Photos were taken 10 days post inoculation from the International Center for Tropical Agriculture, Colombia. 
isolate. The disease indices could not be compared between locations by analysis of variance because different sheath blight isolates were used due to international quarantine restrictions. Within each location, the significant differences among rice cultivars were compared between the means of disease index using Fisher's least significant difference test at $P=0.05$ (STATGRAPHICS Centurion, ver.15.0).

\section{RESULTS}

Uniform inoculum PDA plugs of actively growing mycelia of $R$. solani from the periphery of the fungal culture resulted in rapid infection and also increased reproducibility of the cultivar response to the pathogen. At all locations, the microchamber created a high-humidity environment and suitable temperature for infection to occur. In the summer, chambers were removed when greenhouse temperatures were above $40^{\circ} \mathrm{C}$ until temperature dropped. In the winter, disease progress was delayed 2 to 7 days and, consequently, disease reactions were evaluated 2 to 7 days later.

The standard cvs. Jasmine 85 and Lemont produced consistently moderately resistant (index 1.4 to 2.9 ) and susceptible (index 5.9 to 8.9 ) reactions, respectively, across the three locations using different $R$. solani isolates in the micro-chamber method (Figs. 1 and 2). In all locations, Pecos was moderately resistant and Rosemont was susceptible. TeQing, known for its moderately resistant reaction in the field $(7,11)$, was more resistant in Texas and Colombia than in Arkansas. Cvs. Cocodrie and Cypress, known for their susceptible field reactions (5), tended to be more susceptible at the Arkansas location than in Texas and Colombia (Figs. 1 and 2).

Field experiments in Arkansas and Texas verified the pathogenicity of $R$. solani isolates used in different locations and also confirmed the results of disease reaction of each test cultivar to R. solani (Fig. 3 ). The standard cv. Jasmine 85 was moderately resistant and Lemont was susceptible. In both locations, TeQing and Pecos were resistant whereas Rosemont and Cocodrie were susceptible. Cypress appeared to be more susceptible in Texas field plots than in Arkansas. Disease reactions determined in the field were consistent with reactions found using the micro-chamber method.

\section{DISCUSSION}

An efficient screening method first observed in Bangladesh was modified to determine cultivar disease responses using adapted germplasm and local $R$. solani isolates. The ability to examine disease reactions to $R$. solani in the greenhouse is a significant improvement over past field methods in terms of developing rapid, efficient response data for breeding and genetics studies. Although the method did

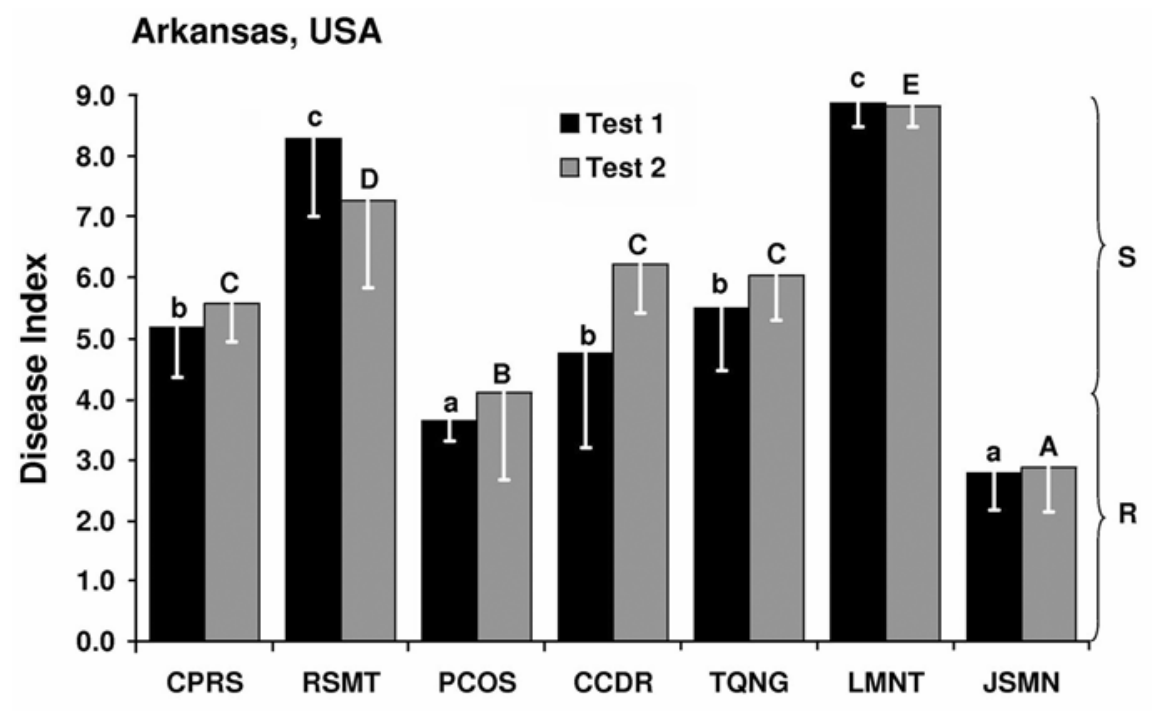

Texas, USA

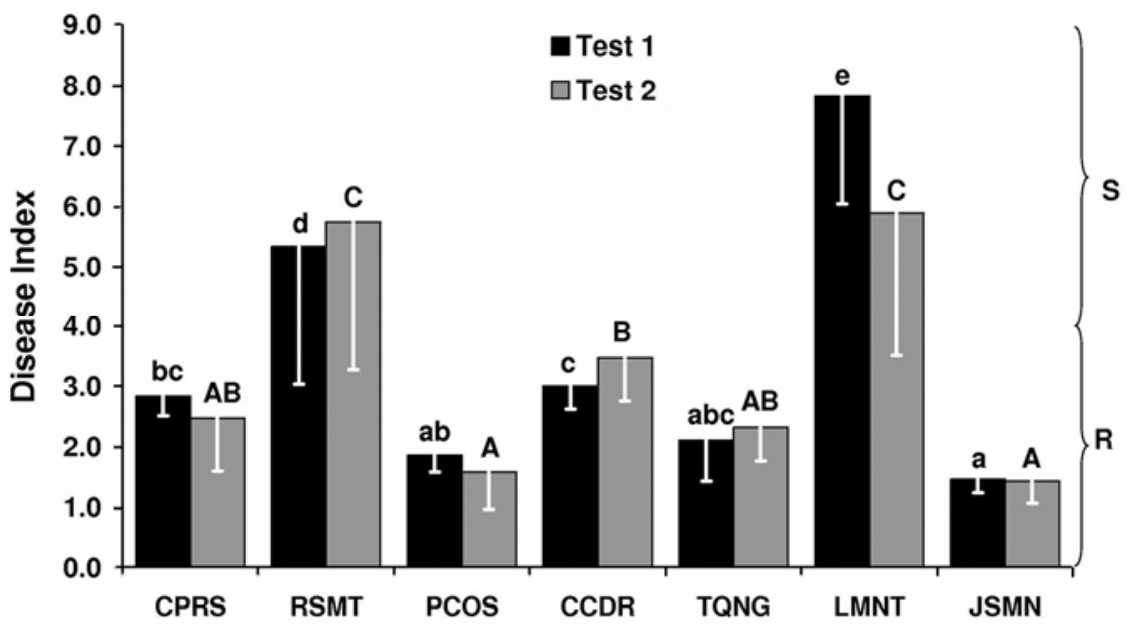

Cali, Colombia

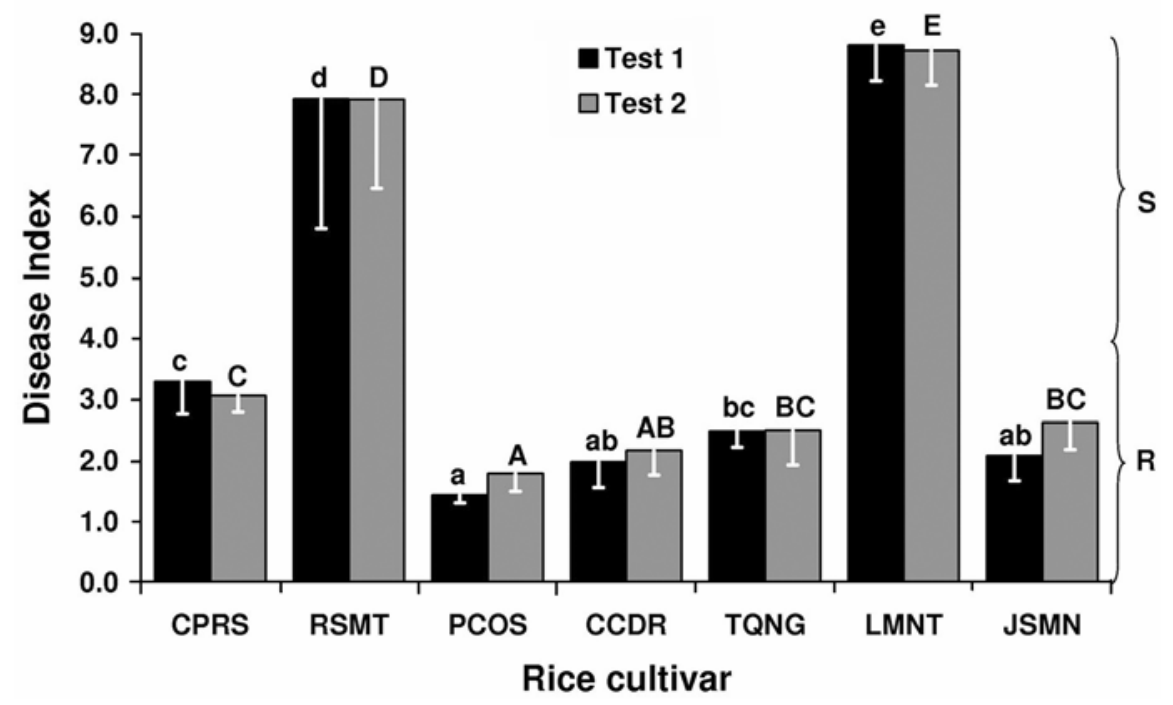

Fig. 2. Sheath blight disease index on rice cultivars inoculated with Rhizoctonia solani inocula in micro-chambers in Arkansas and Texas, United States, and Cali, Colombia. Test 2 is a complete sequential rerun of test 1 . Within a test, the bar means topped with the same letters do not significantly differ at 5\% level (index was determined as lesion height/plant height $\times 9$ ). CPRS = Cypress, RSMT = Rosemont, PCOS $=$ Pecos, CCDR = Cocodrie, TQNG = TeQing, LMNT = Lemont, and JSMN = Jasmine 85 . 
not prove to be $100 \%$ reliable, it was as consistent and reliable as field plot data. An efficient screening method first observed in Bangladesh was modified to determine cultivar responses using adapted germplasm and local $R$. solani isolates. Overall, the greenhouse results ranked the cultivars in the same order, from susceptible to moderate to resistant, as did the field observations, which were themselves consistent with past field observations. Lemont and Rosemont proved to be highly susceptible across all field and greenhouse tests, whereas Jasmine 85 and Pecos were the most resistant. The difference in susceptibility observed between the field and micro-chamber experiments and between the locations for cvs. Cocodrie, Cypress, and TeQing may be due to different resistance mechanisms or gene expressions in the seedling versus mature growth stages. For example, Cocodrie exhibited a susceptible reaction in Colombia when inoculated plants were 50 days old (data not shown). There also could be different genotypic responses to local sheath blight isolates.

Obvious challenges lie ahead in quantifying minor variations of resistance to the sheath blight pathogen that are expected to be observed in traits under polygenic control. Published methods for screening for sheath blight resistance do not completely rule out other variables that often influence disease response, such as activity of mycelia, use of sclerotia, and plant morphology $(2,3,10,14-16)$. A series of methods previously were investigated at the International Rice Research Institute (IRRI) with the aim of screening cultivars in less time and saving space. One method involved inoculation of detached leaves with sclerotia
(10). Although sclerotia are the natural source of inoculum, they often produce inconsistent results using detached leaf inoculation protocol (P. Singh and Y. Jia, unpublished data). Variable results from using sclerotia may be due to the fact that these are primarily resting structures of the pathogen, isolates vary in the size of sclerotia that are produced, and differences in germination of sclerotia within a single isolate has been observed commonly (Y. Wamishe and Y. Jia, unpublished data).

In the past, IRRI developed a standard evaluation system for rice sheath blight disease based on relative lesion length, which is the average vertical height of the uppermost lesion expressed as a percentage of the average plant height (4). Evaluating seedlings in micro-chambers may reduce confounding effects of such factors as plant height and plant maturity, which may obscure evidence of the actual disease response of a genotype $(7,11)$. In addition, the current study also demonstrated the importance of using a standardized inoculation procedure. For example, plants initially were inoculated with 3-, 6-, and 10day-old cultures in preliminary tests, and it was observed that 3-day-old cultures produced the most reproducible infections.

Although $R$. solani is considered a major pathogen of rice around the world, identifying genetic sources of resistance has been hindered by the inability to have an efficient and effective screening method. Development of a robust screening system for detecting differential host responses to $R$. solani in the greenhouse opens an opportunity to collect data year round, and significantly enhances the ability to identify genetic sources and breeding

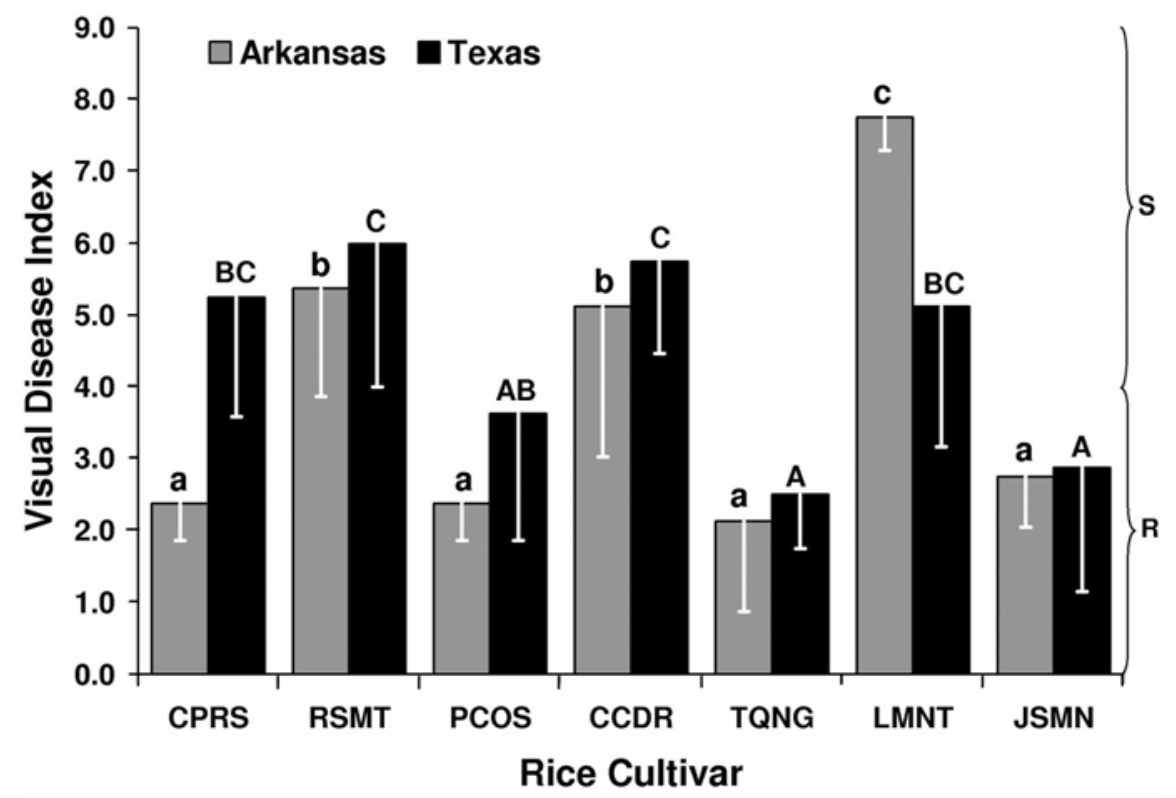

Fig. 3. Visual sheath blight scores of rice cultivars to Rhizoctonia solani under field conditions in Arkansas and Texas, United States. Within each location, the bar means topped with the same letters do not significantly differ at the $5 \%$ level. The score of the rice cultivar was based on a 0 -to- 9 scale. CPRS $=$ Cypress, $\mathrm{RSMT}=$ Rosemont, $\mathrm{PCOS}=$ Pecos, $\mathrm{CCDR}=$ Cocodrie, $\mathrm{TQNG}=$ TeQing, $\mathrm{LMNT}=\mathrm{Le}-$ mont, and JSMN = Jasmine 85 . progeny resistant to sheath blight disease. Similar to experiments conducted at Zhou et al. (17), speed of mycelial growth and lesion length were used to infer genetic response to the pathogen in this study. Consistency in cultivar responses indicated that the micro-chamber method is able to predict the overall field performance of rice cultivars. The micro-chamber method for sheath blight evaluation has the following advantages over inoculated field nurseries. (i) The entire test requires less than 30 days to complete, which will shorten the time needed for screening for genetic resistance. (ii) The experiment requires relatively little space compared with a field trial, numerous replications can be evaluated per experiment, and the variability between replicates is less than in field tests. (iii) Unlike field experiments that are limited to a growing season, greenhouse studies extend the time available for the evaluation of disease reactions and allow increased replications. (iv) Only 5 to $10 \mathrm{~g}$ of seed are required for a highly replicated experiment facilitating evaluation at earlier generations in a breeding program. And, (v) since ratings are done on individual plants rather than bulk-seeded field plots, scoring segregating breeding material or mapping populations is more accurate. Even though there are these advantages, it remains to be determined if this method can distinguish moderate levels of resistance as would be expected in a segregating populations used to identify quantitative trait loci controlling resistance to the pathogen.

$R$. solani is a widespread pathogen causing disease on a broad host range; therefore, the micro-chamber method used in this study may be suitable for evaluating disease reactions of a number of agronomically important crops.

\section{ACKNOWLEDGMENTS}

The project was supported in part by the USDA Cooperative State Research, Education, and Extension Service-National Research Initiative-Applied Plant Genomics Program entitled "RiceCAP: A coordinated research, education, and extension project for the application of genomic discoveries to improve rice in the United States" (USDA/SCREES grant 204-35317-14867). The China National Natural Science Foundation supported J. Xie's visit to DB NRRC in cooperation with the University of Arkansas Rice Research and Extension Center, and U.S. FAO supported S. Pinson's visit to BRRI. We thank A. Ali (BRRI), for expertise and providing the initial idea of using a micro-chamber method in Bangladesh; J. Oard, for participation in the test; M. Lin, T. Beaty, K. Gubrij, and P. Roberts for excellent technical assistance;. And F. Lee, C. Rush, and D. Groth for expertise in field experiments.

\section{LITERATURE CITED}

1. Cartwright, R. D., Parsons, P. E., Sutton, E. A. and Lee, F. N. 2002. Disease monitoring and evaluation of rice cultivars on Arkansas farms Pages 219-228 in: B. R. Wells Rice Research Studies 2002. R. J. Norman and J. F. Meullenet, eds. University of Arkansas Agriculture Experiment Station Research, Fayetteville, AR. 
2. Eizenga, G. C., Lee, F. N., and Rutger, J. N. 2002. Screening Oryza species plants for rice sheath blight resistance. Plant Dis. 86:808-812.

3. Gangopadhyay, S., and Chakraborty, N. 1982. Sheath blight of rice. Rev. Plant Pathol. 61:451-455.

4. IRRI. 2002. Standard evaluation system for rice (SES). International Rice Research Institute, Manila, Philippines.

5. Lee, F. N., Moldenhauer, K. A. K., Gibbons, J. W., Emerson, M. J., and Clark, S. D. 2002. Rice blast and sheath blight evaluation results for the 2002 uniform regional rice nursery. Pages 73-84 in: B. R. Wells Rice Research Studies 2002. R. J. Norman and J. F. Meullenet, eds. University of Arkansas Agriculture Experiment Station Research.

6. Lee, F. N., and Rush, M. C. 1983. Rice sheath blight: a major rice disease. Plant Dis. 67:829832 .

7. Li, Z., Pinson, S. R. M., Marchetti, M. A., Stansel, J. W., and Park, W. D. 1995. Characterization of quantitative trait loci (QTLs) in cultivated rice contributing to field resistance to sheath blight (Rhizoctonia solani). Theor.
Appl. Genet. 91:382-388.

8. Marchetti, M. A. 1983. Potential impact of sheath blight on yield and milling quality of short-stature rice lines in the southern United States. Plant Dis. 67:162-165.

9. Marchetti, M. A., and Bollich, C. N. 1991. Quantification of the relationship between sheath blight severity and yield loss in rice. Plant Dis. 75:773-775.

10. Ou, S. H. 1985. Rice Diseases. Commonwealth Mycological Institute, Kew, England.

11. Pinson, S. R. M., Capdevielle, F. M., and Oard, J. H. 2005. New and confirmed QTLs conditioning sheath blight resistance in rice using recombinant inbred lines. Crop Sci. 45:503-510.

12. Rush, M. C., and Lee, F. N. 1992. Sheath blight. Pages 22-23 in: Compendium of Rice Diseases. R. K. Webster, and P. S. Gunnell, eds. The American Phytopathological Society, St. Paul, MN

13. Savary, S., Castilla, N. P., Elazegui, F. A., McLaren, C. G., Ynalvez, M. A., and Teng, P. S. 1995. Direct and indirect effects of nitrogen supply and disease source structure on rice sheath blight spread. Phytopathol- ogy 85:959-965.

14. Singh, A., Rohilla, R., Singh, U. S., Savary, S. Willocquet, L., and Duveiller, E. 2002. An improved inoculation technique for sheath blight of rice caused by Rhizoctonia solani. Can. J. Plant Pathol. 24:65-68.

15. Wasano, K., Oro, S., and Kido, Y. 1983. The syringe inoculation method for selecting rice plants resistant to sheath blight, Rhizoctonia solani Kühn. Jpn. J. Trop. Agric. 27:131-134.

16. Yoshimura, S., and Nishizawa, T. 1954. Studies on method of testing varietal resistance of upland rice plants caused by sheath blight, Hypnochus sasaki Shirai. Bull. Kyushu Agric. Exp. Stn. 2:361-376.

17. Zhou, E. X., and Yang, M. 1999. Pathogenicity and anastomosis of rice sheath blight pathogen in Guangdong Province. Guangdong Agric. Sci. 5:36-38. (English abstract, in Chinese)

18. Zou, J. H., Pan, X. B., Chen, Z. X., Xu, J. Y., Lu, J. F., Zhai, W. X., and Zhu, L. H. 2000. Mapping quantitative trait loci controlling sheath blight resistance in two rice cultivars (Oryza sativa L.). Theor. Appl. Genet. 101:569-573 